\title{
Lower skeletal muscle mass on CT body composition analysis is associated with adverse clinical course and outcome in children with COVID-19
}

\author{
Rida Salman ${ }^{1}$ (D) Marla B. Sammer ${ }^{1} \cdot$ Bettina L. Serrallach ${ }^{1} \cdot$ Haleh Sangi-Haghpeykar ${ }^{2} \cdot$ Ananth V. Annapragada $^{1}$. \\ R. Paul Guillerman ${ }^{1}$
}

Received: 5 July 2021 / Accepted: 21 January 2022 / Published online: 21 February 2022

(C) Italian Society of Medical Radiology 2022

\begin{abstract}
Objectives To assess the association between body composition measures in children with COVID-19 and severity of the disease course and clinical outcome.

Methods A retrospective study of children (<19 years) with COVID-19 admitted to the hospital who underwent CT of the chest and/or abdomen was conducted. Data compiled from electronic medical records included demographics, body mass index (BMI), length of stay, ICU admission, invasive mechanical ventilation and death. Waist circumference and perimeters for skeletal muscle mass (abdominal, psoas and paraspinal muscles) were measured on an axial CT image at the level of the twelfth thoracic vertebra or first lumbar vertebra using FIJI software.

Results Fifty-seven subjects were identified (54\% male, median age 15.6 years, 61\% Hispanic, 23\% African-American). $25 \%(14 / 57)$ were admitted to the ICU and 21\% (12/57) needed intubation. 9\% (5/57) died. Waist circumference ranged between 53.2 and $138.4 \mathrm{~cm}$ (mean $86.58 \pm 18.74 \mathrm{~cm}$ ) and skeletal muscle mass ranged between 0.6 and $6.8 \mathrm{~cm}^{2}$ (mean $3.5 \pm 1.19 \mathrm{~cm}^{2}$ ). Lower skeletal muscle mass had a univariate association with ICU admission (odds ratio (OR) $0.4 ; 95 \% \mathrm{CI}$ $0.17-0.76 ; p=0.01$ ) and mortality (OR $0.22 ; 95 \%$ CI $0.04-0.69 ; p=0.01)$. Multivariate analysis showed similar association after controlling for comorbidities (adjusted OR 0.46; 95\% CI 0.19-0.95; $p=0.04$ and adjusted OR 0.31; 95\%CI 0.06-0.95; $p=0.04$, respectively). There was no association between BMI or waist circumference with ICU stay, mechanical ventilation or mortality.

Conclusion Lower skeletal muscle mass is associated with an adverse clinical course and outcome in children with COVID-19.
\end{abstract}

Keywords COVID-19 $\cdot$ Skeletal muscle mass $\cdot$ Body composition $\cdot$ Obesity

$\begin{array}{ll}\text { Abbreviations } \\ \text { CDC } & \text { Centers for Disease Control and Prevention } \\ \text { BMI } & \text { Body mass index } \\ \text { CT } & \text { Computed tomography } \\ \text { ICU } & \text { Intensive care unit } \\ \text { RT-PCR } & \text { Real-time reverse transcriptase }\end{array}$

Rida Salman

rxsalman@texaschildrens.org

1 Edward B. Singleton Department of Radiology, Texas Children's Hospital, Baylor College of Medicine, 6701 Fannin St., Suite 470, Houston, TX, USA

2 Department of Obstetrics and Gynecology, Baylor College of Medicine, One Baylor Plaza, Houston, TX 77030, USA
MIS-C Multisystem inflammatory syndrome in children

T12 Twelfth thoracic vertebra

L1 First lumbar vertebra

SD Standard deviation

IQR Interquartile range

OR Odds ratio

CI Confidence interval

NK Natural killer

MRI Magnetic resonance imaging 


\section{Introduction}

The first pediatric case of COVID-19, the disease caused by the SARS-CoV-2 virus, in the USA was reported to Centers for Disease Control and Prevention (CDC) on March 2, 2020 [1]. Since then, comprehensive clinical data collection has been initiated to determine risk factors for severe pediatric COVID-19 course and poor outcomes. Obesity, measured as body mass index (BMI), was found to be among the factors that increase the risk of children for severe COVID-19 illness [2]. Childhood and adolescent obesity is considered a public health concern in the USA with a prevalence of $18.5 \%$ in 2015-2016 and a steady rise in prevalence since 2000 [3].

Body composition, including the amount and relative proportion of skeletal muscle and adipose tissue, cannot be differentiated from body weight by the simple measure of BMI. Cross-sectional imaging modalities, including computed tomography (CT), can be used to assess anthropometric variables such as waist circumference, skeletal muscle and visceral and subcutaneous adipose tissue $[4,5]$. Furthermore, as the data needed for these variables are already available on imaging performed for other purposes, the use of automated body composition to potentially predict clinical outcomes shows promise in the rapidly evolving field of predictive analytics [6]. Studies of adult COVID-19 patients investigating the correlation between these anthropometric variables and COVID-19 disease course and outcome revealed an increased need for intensive care unit (ICU) treatment and mechanical ventilation in patients with a higher ratio of waist circumference per paravertebral muscle circumference (FMR) [7] and higher values of visceral adipose tissue and upper abdominal circumference $[8,9]$. The value of body composition in the clinical course and outcome of pediatric COVID-19 patients has not yet been assessed. Therefore, we studied the relationship between waist circumference and skeletal muscle mass of COVID-19 positive children measured on chest and/or abdominal CT scans and the clinical course and outcome including ICU admission, invasive mechanical ventilation and death.

\section{Materials and methods}

Our institutional review board approved this retrospective study, and patient consent was waived.

\section{Patients' demographics}

The study included children under 19 years of age who tested positive for SARS-CoV-2 by real-time reverse transcriptase (RT-PCR) analysis of nasopharyngeal swab samples between March and September 2020, were admitted to the hospital and underwent CT of the chest and/or abdomen. Children diagnosed with multisystem inflammatory syndrome in children (MIS-C) were excluded.

\section{Clinical data}

Data collected from electronic medical records included demographics, vital signs, BMI, reason for hospital admission, date and result of SARS-CoV-2 testing, date and result of the CT scan, hospital stay duration and course including need for ICU stay and mechanical ventilation, comorbidities and clinical outcome. BMI was categorized as normal versus abnormal (overweight or obese) using the CDC age- and sex-specific definitions of $85-95^{\text {th }}$ percentile for overweight and 95 th percentile or greater for obesity.

\section{CT acquisition parameters}

The CT examinations were performed either on a 320 row multidetector computed tomography (MDCT) scanner (Aquilion ONE, Canon Medical Systems, Tochigi, Japan) or a $192 \times 2$ slice dual-source CT (DSCT) scanner (Somatom Force, Siemens Medical Solutions, Forchheim, Germany). Tube voltage was set at $80-120 \mathrm{kV}$ and tube current at $40-100 \mathrm{~mA}$ according to the patients' age. Automated tube-current modulation was used with the dual-source CT. The contrast agent used was Omnipaque $300 \mathrm{mg} / \mathrm{mL}$ (GE Healthcare, Marlborough, Massachusetts, USA).

For non-gated CT chest, abdomen and pelvis with contrast, $1.5-2 \mathrm{~mL} / \mathrm{Kg}$ of contrast was administered with an injection rate of $0.5-1.5 \mathrm{~mL} / \mathrm{sec}$ depending on catheter size and patient size. Scan coverage was from just above the lung apices to just below the symphysis pubis.

For chest CT for suspected pulmonary embolism, $2-2.5 \mathrm{~mL} / \mathrm{Kg}$ of contrast is administered with an injection rate of $2.5-5 \mathrm{~mL} / \mathrm{sec}$ depending on catheter size and patient size. Scan coverage was from just above the lung apices to just below the diaphragm. On the Canon Aquilion One scanner, a volumetric gated scan mode was used for z-axis coverage of less than $16 \mathrm{~cm}$ and a helical protocol was used for a $\mathrm{z}$-axis coverage greater than $16 \mathrm{~m}$. Turbo Flash mode was used with the Siemens Somatom Force dual-source scanner.

\section{Image analysis}

Waist circumference and metrics of skeletal muscle mass (abdominal muscles including the rectus abdominis, external and internal oblique and transverse abdominis, psoas and paraspinal muscles including the quadratus lumborum and erector spinae) were measured by two authors (RS and BS) on an axial CT image at the level of the twelfth thoracic vertebra (T12) or first lumbar vertebra (L1) using FIJI [10] 
which is an open source image processing package based on Image J [11]. These vertebral levels were selected to maximize the number of CT scans with useful data since these levels are generally included on both chest and abdomen CT scans. The T12 vertebra was used for measurements when the L1 vertebra was not included on chest CT $(n=4)$.

Patients $(n=3)$ whose CT quality was not suitable for analysis on ImageJ software due to artifact or a restricted field of view for waist circumference or skeletal muscle mass measurement were excluded. The measurement technique is detailed in a previously published article by Gomez et al. [11] and summarized in Fig. 1.

The following formulas [11] were applied:

For waist circumference $(\mathrm{cm})$ : (perimeter, $\mathrm{mm}$ ) divided by 10 .
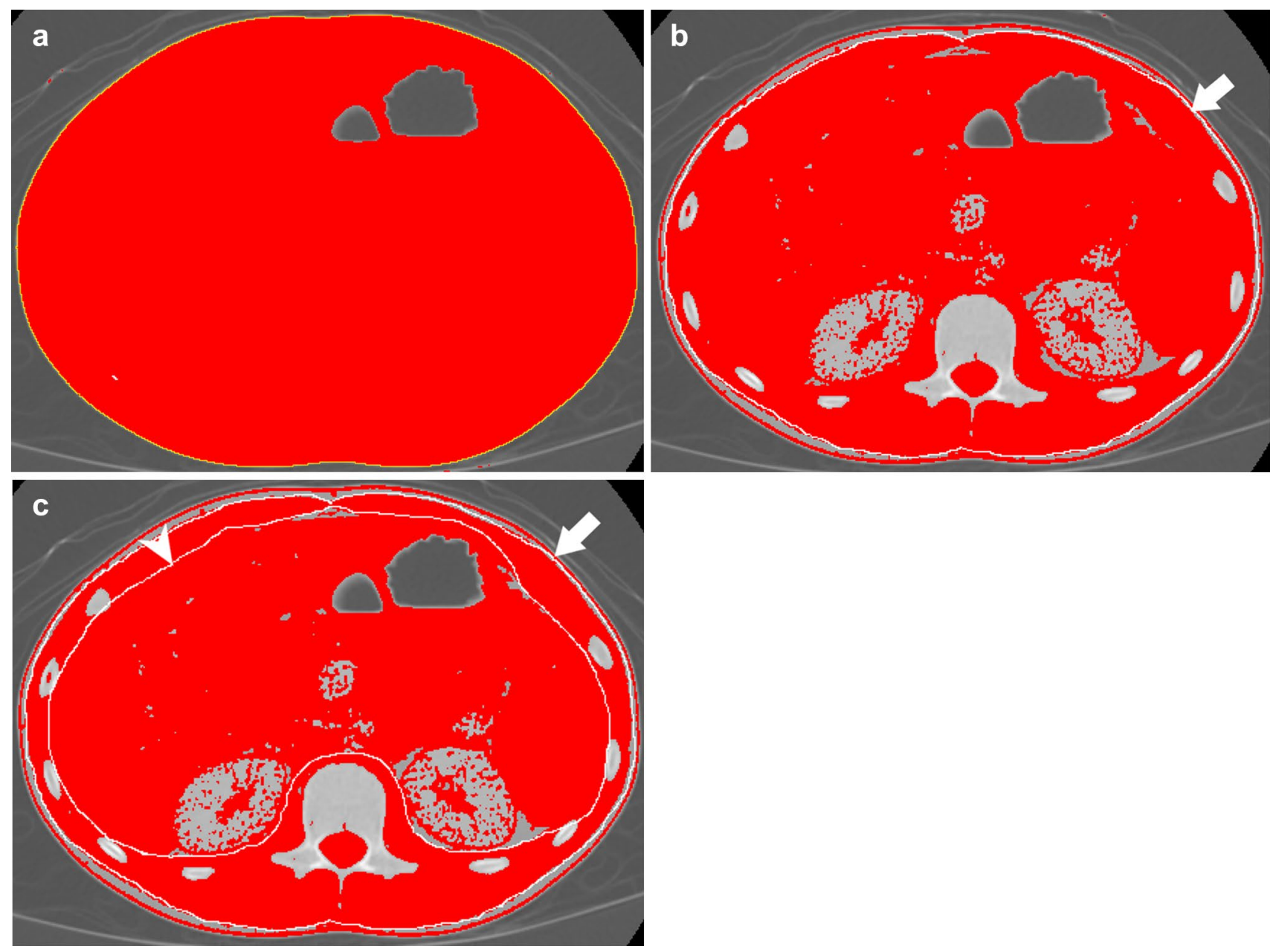

Fig. 1 Measurement technique summarized in three steps. Step 1: after adjusting the image threshold between -250 and +1000 , we click on the outer surface of the threshold-highlighted area with a wand tracing tool which delineates the abdominal perimeter (yellow contour in 1a). Step 2: the image returns to its original gray color after adjusting the threshold between -250 and -250 and we remove the yellow line by clicking on freehands selection tool. A stylus is
For skeletal muscle mass $\left(\mathrm{cm}^{2}\right)$ : (outer muscle area - inner muscle area, $\mathrm{mm}^{2}$ ) divided by 100 .

\section{Statistical analysis}

All statistical analyses were conducted with SAS software (version 9.4, Cary, North Carolina, USA). All variables were assessed for normality. Continuous variables with normal distribution were specified as mean \pm standard deviation (SD), otherwise as median and interquartile range (IQR). The endpoints were ICU admission, mechanical ventilation and death. Univariate and multivariate logistic regression models were conducted to analyze the relationship between these endpoints as dependent variables and BMI, waist circumference and skeletal muscle mass as predictors and age,

then used to trace the outer muscle perimeter (arrow in $1 \mathrm{~b}$ and $1 \mathrm{c}$ ) for which measurement is obtained after making the threshold between -29 and +150 . Step 3: the threshold is adjusted back between -250 and -250 then tracing of the inner muscle perimeter (arrowhead in 1c) is performed using the stylus. This measurement is also obtained after adjusting the threshold between -29 and +150 
sex, ethnicity, race and comorbidities as possible covariates. BMI was also used as covariate in the analysis. Odds ratio (OR) and $95 \%$ confidence interval $(\mathrm{CI})$ were calculated. Pearson's correlation (r) was also used to measure the association between body composition measurements and mechanical ventilation duration. Statistical significance was defined as $p \leq 0.05$.

The inter-rater reliability or agreement between the two authors who performed the measurements was assessed using the intraclass correlation coefficient (ICC) with values less than $0.5,0.5-0.75,0.75-0.9$ and greater than 0.9 indicating poor, moderate, good and excellent reliability, respectively.

\section{Results}

\section{Patients' demographic and clinical data}

Fifty-seven patients (54\% male) met inclusion criteria with a median (IQR) age of 15.6 (9.4-17.2). 61\% were Hispanic and $23 \%$ were African-American. Thirty patients $(55 \%)$ had abnormal BMI; specifically, 13 of whom were overweight and 17 of whom were obese. Of these 57 patients, $25 \%$ $(14 / 57)$ were admitted to the ICU, 21\% (12/62) needed intubation and 9\% (5/57) died. Twenty-four patients (42\%) had comorbidities including cancer $(n=10)$, chronic lung disease related to prematurity $(n=4)$, sickle cell disease $(n=3)$ and others $(n=7)$. Table 1 summarizes the demographic and clinical characteristics.

\section{Indications for obtained CT}

The main indication for abdominal CT was the presence of gastrointestinal signs and symptoms including abdominal pain, vomiting, diarrhea and abdominal distention with either equivocal ultrasound findings (e.g., for appendicitis) or limited abdominal ultrasound due to patient's body habitus.

The main indication for CT chest was possible pulmonary embolism on the basis of chest pain, cough or tachypnea.

\section{Image analysis}

Waist circumference ranged between 53.2 and $138.4 \mathrm{~cm}$ (mean $86.58 \pm 18.74 \mathrm{~cm}$ ). As expected, no difference between the two raters was noted since this step is automated. Skeletal muscle mass ranged between 0.6 and 6.8 $\mathrm{cm}^{2}$ (mean $3.5 \pm 1.19 \mathrm{~cm}^{2}$ ) in both raters with excellent interrater agreement; the average measure ICC was 0.969 with a 95\% confidence interval from 0.947 to 0.982 .

There was a statistically significant association between the presence of comorbidities and the clinical course and
Table 1 Demographic, clinical and radiological characteristics of the study population

\begin{tabular}{ll}
\hline Characteristic & All patients $(n=62)$ \\
\hline Demographic and clinical characteristics & \\
Age (years), median (IQR) & $15.6(9.4-17.2)$ \\
Male sex N (\%) & $31(54)$ \\
African-American race N (\%) & $13(23)$ \\
Hispanic ethnicity N (\%) & $35(61)$ \\
Abnormal body mass index (kg/m $\left.{ }^{2}\right) \mathrm{N}(\%)$ & $30(55)$ \\
Temperature (F), median (IQR) & $98.2(97.7-98.7)$ \\
Systolic blood pressure (mmHg), mean $\pm \mathrm{SD}$ & $114 \pm 12.98$ \\
Diastolic blood pressure (mmHg), mean $\pm \mathrm{SD}$ & $65 \pm 10.42$ \\
Heart rate (beats per minute), median (IQR) & $87(73-101)$ \\
Respiratory rate (breath per minute), median & $18(16-22)$ \\
$\quad$ IQR) & \\
Comorbidities N (\%) & $24(42)$ \\
Intensive care unit admission N (\%) & $14(25)$ \\
Mechanical ventilation N (\%) & $12(21)$ \\
Mortality N (\%) & $5(9)$ \\
Radiological characteristics & \\
Waist circumference (cm), mean $\pm \mathrm{SD}$ & $86.58 \pm 18.74$ \\
Skeletal muscle mass (cm $\left.{ }^{2}\right)$, mean $\pm \mathrm{SD}$ & $3.5 \pm 1.19$ \\
\hline
\end{tabular}

*Only 55 patients had documented weight and height on charts. Abnormal body mass index includes overweight (13 patients) and obese (17 patients) categories. The remaining characteristics were documented for 57 patients

$S D$ standard deviation; $N$ number

outcome (Table 2). Lower skeletal muscle mass had a univariate association (Table 3 ) with ICU admission (OR $0.4 ; 95 \%$ CI $0.17-0.76 ; p=0.01)$ and mortality (OR 0.22 ; 95\% CI 0.04-0.69; $p=0.01$ ). Multivariate analysis (Table 4) showed similar association after controlling for the presence of comorbidities (adjusted OR 0.46; 95\%CI 0.19-0.95; $p=0.04$ and adjusted OR $0.31 ; 95 \%$ CI $0.06-0.95 ; p=0.04$, respectively).

There was no statistically significant association between BMI or waist circumference with ICU stay, mechanical ventilation or mortality. None of the body composition measures showed a statistically significant correlation with mechanical ventilation duration (Figs. 2 and 3). Two cases are presented in Figs. 4 and 5.

\section{Discussion}

Obesity is a comorbidity that has been reported to influence the course and outcome of children affected by SARSCoV-2, including ICU admission and mechanical ventilation $[2,12,13]$. In these studies, obesity was defined based on BMI which does not take body composition into consideration [14]. CT-based measurements of skeletal muscle and 
Table 2 Demographic and clinical characteristics and patient's clinical course and outcome. Only the presence of comorbidities was found to be statistically significantly correlated

\begin{tabular}{|c|c|c|c|c|c|c|c|c|c|c|}
\hline & & \multicolumn{3}{|c|}{ Intensive care unit } & \multicolumn{3}{|c|}{ Mechanical ventilation } & \multicolumn{3}{|l|}{ Mortality } \\
\hline & & Yes & No & $P$ value & Yes & No & $P$ value & Yes & No & $P$ value \\
\hline \multicolumn{2}{|l|}{ Age, mean (SD) } & $10.5(6.5)$ & $13.8(4.5)$ & 0.07 & $12.2(7)$ & $13.2(4.6)$ & 0.9 & $10.1(7.6)$ & $13.3(4.9)$ & 0.44 \\
\hline \multirow[t]{2}{*}{ Sex, N (\%) } & Female & $5(36)$ & $21(49)$ & 0.39 & $5(42)$ & $21(47)$ & 0.75 & $3(60)$ & $23(44)$ & 0.49 \\
\hline & Male & $9(64)$ & $22(51)$ & & $7(58)$ & $24(53)$ & & $2(40)$ & $29(56)$ & \\
\hline \multirow[t]{2}{*}{ Ethnicity, N (\%) } & Hispanic & $6(43)$ & $29(67)$ & 0.1 & $5(42)$ & $30(67)$ & 0.11 & $4(80)$ & $31(60)$ & 0.64 \\
\hline & Other & $8(57)$ & $14(33)$ & & $7(58)$ & $15(33)$ & & $1(20)$ & $21(40)$ & \\
\hline \multirow[t]{2}{*}{ Race, N (\%) } & African-American & $4(29)$ & $9(21)$ & 0.71 & $4(42)$ & $9(20)$ & 0.44 & $0(0)$ & $13(25)$ & 0.58 \\
\hline & Other & $10(71)$ & $34(79)$ & & $8(67)$ & $36(80)$ & & $5(100)$ & $39(75)$ & \\
\hline \multirow[t]{2}{*}{ BMI, N(\%) } & Normal & $6(43)$ & $19(46)$ & 0.82 & $5(42)$ & $20(47)$ & 0.77 & $3(60)$ & $22(44)$ & 0.65 \\
\hline & Abnormal & $8(57)$ & $22(54)$ & & $7(58)$ & $23(53)$ & & $2(40)$ & $28(56)$ & \\
\hline \multirow[t]{2}{*}{ Comorbidity, $\mathrm{N}(\%)$} & Yes & $11(79)$ & $13(30)$ & 0.002 & $9(75)$ & $15(33)$ & 0.02 & $5(100)$ & $19(37)$ & 0.01 \\
\hline & No & $3(21)$ & $30(70)$ & & $3(25)$ & $30(67)$ & & $\mathbf{0}(\mathbf{0})$ & $33(64)$ & \\
\hline
\end{tabular}

$B M I$ body mass index; $N$ number; $S D$ standard deviation; $\%$, percentage; $p$ value for age is based on Wilcoxon rank sum test. Variables with statistically significant results are bolded

Table 3 Univariate logistic regression analyses for ICU admission, mechanical ventilation and mortality. Only skeletal muscle mass was found to be statistically significantly correlated

\begin{tabular}{|c|c|c|c|}
\hline & $\begin{array}{l}\text { Intensive care unit } \\
\text { OR }(95 \% \mathrm{CI}), p \text { value }\end{array}$ & $\begin{array}{l}\text { Mechanical ventilation } \\
\text { OR }(95 \% \mathrm{CI}), p \text { value }\end{array}$ & $\begin{array}{l}\text { Mortality } \\
\text { OR }(95 \% \mathrm{CI}), p \text { value }\end{array}$ \\
\hline Body mass index & $1.15(0.34-4.06), p=0.82$ & $1.22(0.34-4.69), p=0.76$ & $0.52(0.07-3.42), p=0.65$ \\
\hline Waist circumference & $0.97(0.93-1.01), p=0.12$ & $0.99(0.96-1.03), p=0.74$ & $0.97(0.91-1.02), p=0.31$ \\
\hline Skeletal muscle mass & $0.4(0.17-0.76), p=0.01$ & $0.84(0.46-1.45), p=0.55$ & $0.22(0.04-0.69), p=0.01$ \\
\hline
\end{tabular}

OR Odds ratio; $C I$ confidence interval. Variables with statistically significant results are bolded

Table 4 Multivariate logistic regression analyses for ICU admission and mortality after adjustment for comorbidities. Only skeletal muscle mass was found to be statistically significantly correlated

\begin{tabular}{lll}
\hline & Intensive care unit & Mortality \\
& AOR $(95 \%$ CI $), p$ value & $(95 \%$ CI $), p$ value \\
\hline Body mass index & $1.71(0.44-7.3), p=0.51$ & $0.74(0.08-5.49), p=0.99$ \\
Waist circumference & $0.98(0.94-1.02), p=0.35$ & $0.99(0.93-1.04), p=0.45$ \\
Skeletal muscle mass & $\mathbf{0 . 4 6}(\mathbf{0 . 1 9 - 0 . 9 5}), \boldsymbol{p}=\mathbf{0 . 0 4}$ & $\mathbf{0 . 3 1}(\mathbf{0 . 0 6}-\mathbf{0 . 9 5}), \boldsymbol{p}=\mathbf{0 . 0 4}$ \\
\hline
\end{tabular}

AOR Adjusted odds ratio; $C I$ confidence interval. Variables with statistically significant results are bolded

adipose tissue are able to quantify body composition [15, 16]. In our study, we find that lower skeletal muscle mass is associated with increased risk of ICU admission and mortality in children with COVID-19 disease, even after adjusting for the presence of comorbidities.

Low skeletal muscle mass has been reported in pediatric patients to be associated with increased risk of prolonged hospital stay, ICU admission, ventilator dependency, poor growth and postoperative complications in children with perforated appendicitis, acute lymphoblastic leukemia, endstage liver or kidney disease, post-transplant, inflammatory bowel disease and Prader-Willi syndrome [17]. A potential mechanistic basis for the relationship between low skeletal muscle mass and poor clinical course and outcome in our study is that reduced skeletal muscle mass may lead to decreased secretion of myokines or muscle-derived cytokines that play an important role in systemic glucose metabolism and hence leading to insulin resistance that is suggested to promote impaired insulin-mediated anti-inflammatory response of lymphocytes [18, 19]. In addition, the depletion of certain myokines such as interleukin-15 will reduce the activation of natural killer $(\mathrm{NK})$ and $\mathrm{T}$ cells, and 


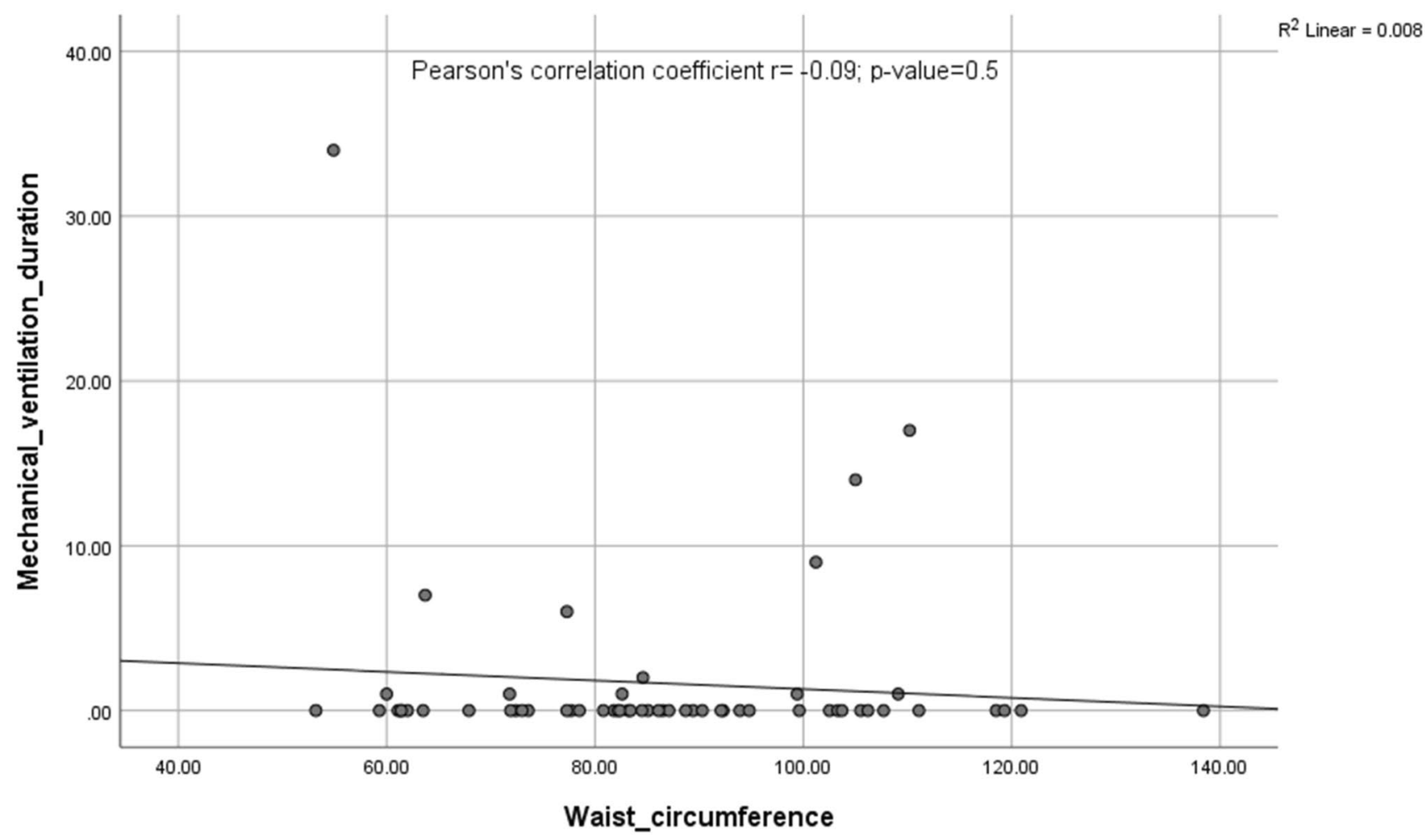

Fig. 2 Pearson's correlation between waist circumference and mechanical ventilation duration. No statistically significant correlation was found with $r=-0.09, p$ value $=0.5$

it has been found that the low NK cell number and skeletal muscle mass predict mortality in the elderly [20]. This defective immune system may be a factor in the greater severity and poor outcomes of COVID-19 disease in patients with lower skeletal muscle mass.

Waist circumference is a surrogate measure of total-body and abdominal fat mass in children [21]. Several studies in adult populations with COVID-19 disease showed that increased waist circumference and visceral adipose tissue are associated with increased disease severity [7-9]. In our cohort, BMI and waist circumference measurements were not shown to be correlated with the clinical course and outcome. However, most of the included children had normal or overweight BMI's, and the number of patients may have been too few to show a statistically significant relation between the waist circumference and disease severity if one exists. Our data suggest it would be useful to screen children with normal or borderline abnormal BMI's for lower skeletal muscle mass.

Among the collected demographic and clinical characteristics including age, sex, ethnicity, race and comorbidities, only the presence of comorbidities showed a statistically significant association with need for ICU admission and mechanical ventilation as well as mortality. More severe infection and outcome were reported among infants and those with comorbidities [1,22]. The five children who died in our cohort had associated comorbidities; four with malignancies and one with history of chronic lung disease related to prematurity and end-stage renal disease. Although the presence of comorbidities can be considered here as a major confounder, the multivariate logistic regression analysis confirmed that lower skeletal muscle mass still predicts the clinical course and outcome of pediatric patients with COVID-19 after adjusting for these comorbidities.

This study has several limitations. First, the study was retrospective, which could limit data collection. However, only two patients lacked an available BMI measurement, and all the remaining queried clinical and radiological data were available. Second, the sample size was relatively small $(n=57)$, although not unexpectedly since the study was conducted in a single institution and limited to pediatric patients. Future prospective and multi-institutional studies could validate our findings and further investigate the pathophysiology. Third, the clinical indications for the CT scans and changes in body composition measures for those with multiple CT scans were not assessed. Body composition measurements can also be obtained from body magnetic resonance imaging (MRI) exams, but the number of body MRI scans in our inclusion group was too small for statistical analysis. Finally, 


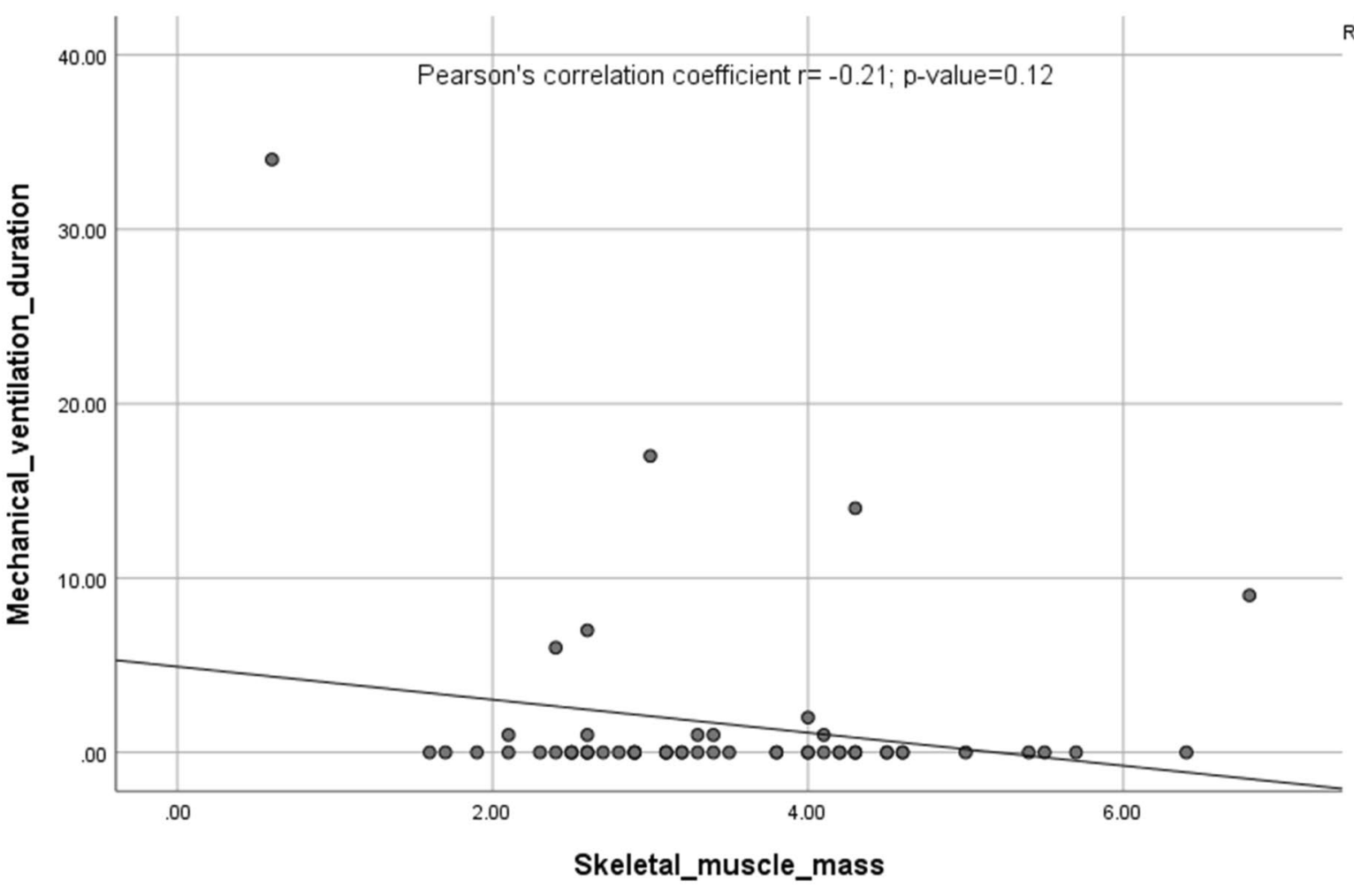

Fig. 3 Pearson's correlation between skeletal muscle mass and mechanical ventilation duration. No statistically significant correlation was found with $r=-0.21, p$ value $=0.12$

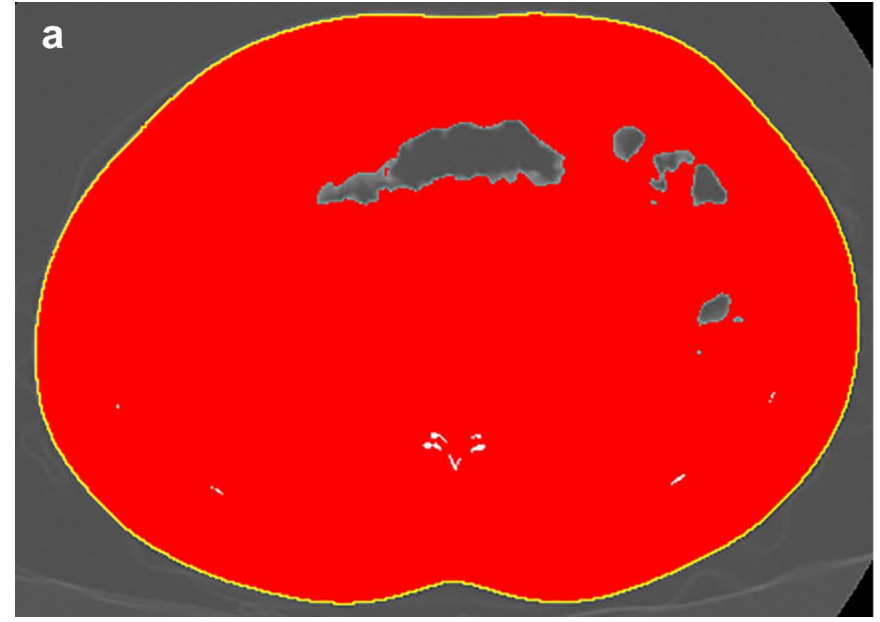

Fig. 4 16-year-old girl with no past medical history presenting with 5-day history of right lower quadrant abdominal pain and nausea. She had abdominal and pelvic ultrasound exams which showed no appendicitis or pelvic pathology. She was admitted for pain control and serial abdominal exams. A CT scan of abdomen and pelvis was obtained due to persistent abdominal pain and showed no acute pathologies. The patient was discharged home after $24 \mathrm{~h}$ with clini-

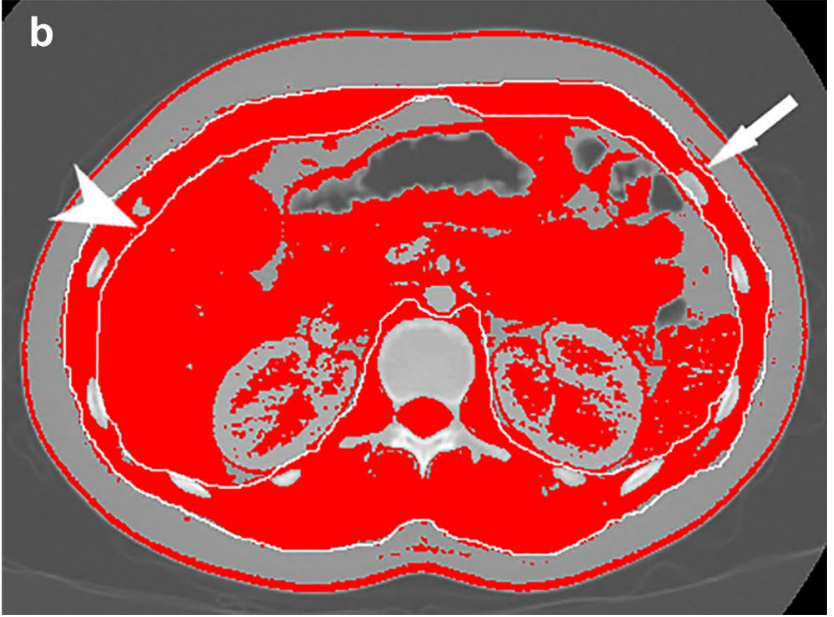

cal improvement. No ICU admission or mechanical ventilation was needed during her short hospital stay. Her BMI was 25.64 (obese for age and sex). Waist circumference measures $86.5 \mathrm{~cm}$ (yellow contour in 1a) and skeletal muscle mass measures $4.5 \mathrm{~cm}^{2}$ (arrow and arrowhead for outer and inner muscle perimeter and area, respectively, in 1b) 


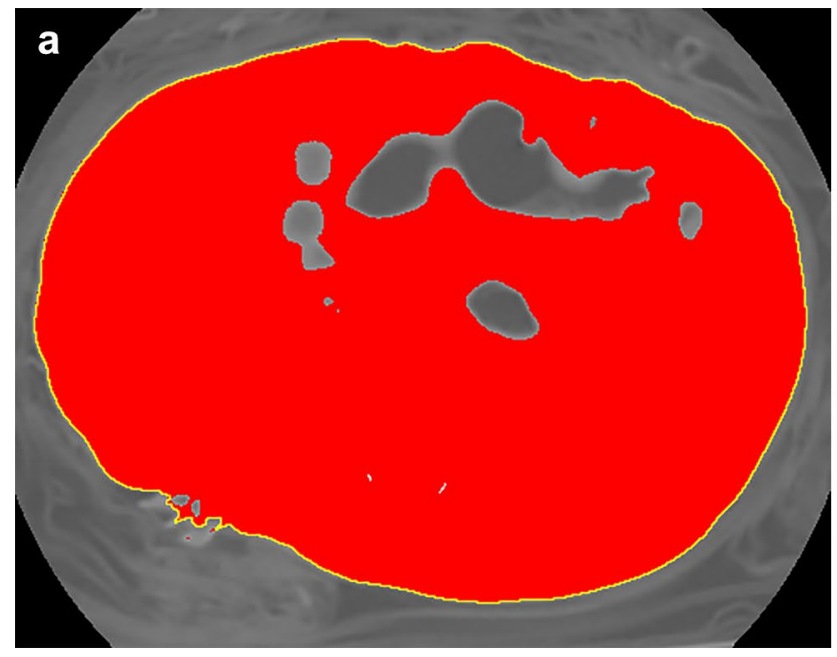

Fig. 5 5-year-old boy with a history of prematurity and hepatoblastoma status post-liver transplantation presenting with abdominal pain and fever. He was found to have cholangitis and was admitted to ICU. The patient eventually died due to sepsis and multiorgan failure. His

in most previous studies, body composition analysis was typically performed on a single axial CT scan at the level of the third lumbar vertebrae. In order to allow broader generalizability to chest CT scans, which are often performed in patients with COVID-19, and do not image the level of L3, we used either the T12 or L1 vertebra in our approach. Of note, past studies have found no significant effect of the measurement site on estimates of adipose tissue and skeletal muscle mass [15, 23]. These limitations notwithstanding, this is the first study to our knowledge investigating the value of body composition analysis on pediatric patients with COVID-19 disease.

In conclusion, lower skeletal muscle mass is associated with an adverse clinical course and outcome in children with COVID-19 and is a promising tool for risk stratification in these patients. Neither BMI nor waist circumference was found to be correlated with outcomes. These findings support the use of quantitative body composition on available imaging (such as CT) for predictive analytics in children with COVID-19 and also suggest that they are more useful than available clinical data such as BMI.

Acknowledgements We acknowledge members of the Texas Children's Hospital COVID-19 Imaging Taskforce, of which Marla B. Sammer, Rida Salman, Ananth V. Annapragada and R. Paul Guillerman were members, but also included Nilesh K. Desai, Thierry A.G.M. Huisman, Prakash M. Masand, Gunes Orman, Victor J. Seghers and Amir H. Pezekhmehr.

Funding The authors state that this work has not received any funding.

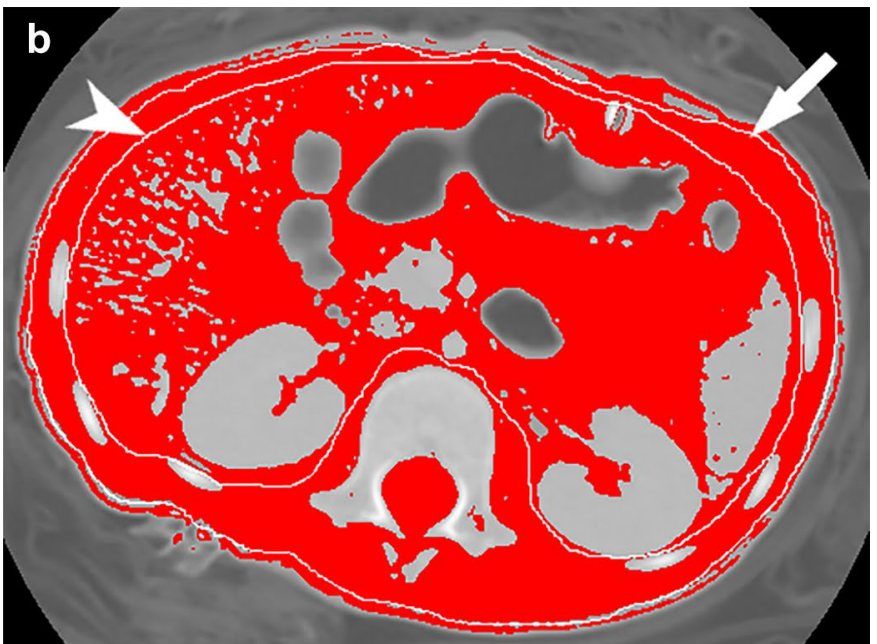

BMI was 15.96 (normal for age and sex). Waist circumference was 53.2 (yellow contour in 2a) and skeletal muscle mass measures 1.6 $\mathrm{cm}^{2}$ (arrow and arrowhead for outer and inner muscle perimeter and area, respectively, in $2 b$ )

\section{Declarations}

Conflict of interest Dr. Ananth Annapragada declares relationships with the following company: Alzeca Inc., Sensulin LLC, stock ownership. The remaining authors of this manuscript declare that they have no conflict of interest.

Data availability Data are available upon request.

Ethical approval Institutional Review Board approval was obtained and written informed consent was waived.

\section{References}

1. Bialek S, Gierke R, Hughes M, McNamara LA, Pilishvili T, Skoff T (2020) Coronavirus disease 2019 in children - United States, February 12-April 2, 2020. MMWR Morb Mortal Wkly Rep 69(14):422-426. https://doi.org/10.15585/mmwr.mm6914e4

2. Shekerdemian LS, Mahmood NR, Wolfe KK et al (2020) Characteristics and outcomes of children with coronavirus disease 2019 (COVID-19) infection admitted to US and Canadian pediatric intensive care units. JAMA Pediatr 174(9):868-873. https://doi. org/10.1001/jamapediatrics.2020.1948

3. Sanyaolu A, Okorie C, Qi X, Locke J, Rehman S (2019) Childhood and adolescent obesity in the United States: a public health concern. Glob Pediatr Health. https://doi.org/10.1177/23337 94X19891305

4. Paris MT (2020) Body composition analysis of computed tomography scans in clinical populations: the role of deep learning. Lifestyle Genomics 13(1):28-31. https://doi.org/10.1159/000503996

5. Paris M, Mourtzakis M (2016) Assessment of skeletal muscle mass in critically ill patients: considerations for the utility of 
computed tomography imaging and ultrasonography. Curr Opin Clin Nutr Metab Care 19(2):125-130

6. Magudia K, Bridge CP, Bay CP et al (2021) Population-scale CTbased body composition analysis of a large outpatient population using deep learning to derive age-, sex-, and race-specific reference curves. Radiology 298(2):319-329. https://doi.org/10.1148/ radiol.2020201640

7. Kottlors J, Zopfs D, Fervers P et al (2020) Body composition on low dose chest CT is a significant predictor of poor clinical outcome in COVID-19 disease - a multicenter feasibility study. Eur Radiol 132:109274. https://doi.org/10.1016/j.ejrad.2020.109274

8. Petersen A, Bressem K, Albrecht J et al (2020) The role of visceral adiposity in the severity of COVID-19: highlights from a unicenter cross-sectional pilot study in Germany. Metabolism 110:154317. https://doi.org/10.1016/j.metabol.2020.154317

9. Watanabe M, Caruso D, Tuccinardi D et al (2020) Visceral fat shows the strongest association with the need of intensive care in patients with COVID-19. Metabolism 111:154319. https://doi. org/10.1016/j.metabol.2020.154319

10. Schindelin J, Arganda-Carreras I, Frise E et al (2012) Fiji: an open-source platform for biological-image analysis. Nat Methods 9(7):676-682. https://doi.org/10.1038/nmeth.2019 (PMID 22743772)

11. Gomez-Perez SL, Haus JM, Sheean P et al (2016) Measuring abdominal circumference and skeletal muscle from a single crosssectional computed tomography image: a step-by-step guide for clinicians using National Institutes of Health ImageJ [published correction appears in JPEN J Parenter Enteral Nutr 40(5), 742-3]. JPEN J Parenter Enteral Nutr 40(3):308-318. https://doi.org/10. $1177 / 0148607115604149$

12. Zachariah P, Johnson CL, Halabi KC et al (2020) Epidemiology, clinical features, and disease severity in patients with coronavirus disease 2019 (COVID-19) in a children's hospital in New York City, New York. JAMA Pediatr 174(10):e202430. https://doi.org/ 10.1001/jamapediatrics.2020.2430

13. Hoang A, Chorath K, Moreira A et al (2020) COVID-19 in 7780 pediatric patients: a systematic review. EClinicalMedicine 24:100433. https://doi.org/10.1016/j.eclinm.2020.100433

14. De Lorenzo A, Romano L, Di Renzo L et al (2019) Triponderal mass index rather than body mass index: an indicator of high adiposity in Italian children and adolescents. Nutrition 60:41-47. https://doi.org/10.1016/j.nut.2018.09.007
15. Derstine BA, Holcombe SA, Ross BE, Wang NC, Su GL, Wang SC (2018) Skeletal muscle cutoff values for sarcopenia diagnosis using T10 to L5 measurements in a healthy US population. Sci Rep 8(1):11369. https://doi.org/10.1038/s41598-018-29825-5

16. Zopfs D, Theurich S, Große Hokamp N et al (2020) Single-slice CT measurements allow for accurate assessment of sarcopenia and body composition. Eur Radiol 30(3):1701-1708. https://doi. org/10.1007/s00330-019-06526-9

17. Ooi PH, Thompson-Hodgetts S, Pritchard-Wiart L, Gilmour SM, Mager DR (2020) Pediatric sarcopenia: a paradigm in the overall definition of malnutrition in children? JPEN J Parenter Enteral Nutr 44(3):407-418. https://doi.org/10.1002/jpen.1681

18. Kwon Y, Jeong SJ (2020) Relative skeletal muscle mass is an important factor in non-alcoholic fatty liver disease in non-obese children and adolescents. J Clin Med 9(10):3355. https://doi.org/ $10.3390 / \mathrm{jcm} 9103355$

19. Villarreal-Calderón JR, Cuéllar RX, Ramos-González MR et al (2019) Interplay between the adaptive immune system and insulin resistance in weight loss induced by bariatric surgery. Oxid Med Cell Longev 2019:3940739. https://doi.org/10.1155/2019/39407 39

20. Lutz CT, Quinn LS (2012) Sarcopenia, obesity, and natural killer cell immune senescence in aging: altered cytokine levels as a common mechanism. Aging (Albany NY) 4(8):535-546. https://doi. org/10.18632/aging.100482

21. Ohta M, Midorikawa T, Hikihara Y et al (2017) Body mass-towaist ratio strongly correlates with skeletal muscle volume in children. PLoS One 12(5):e0177155. https://doi.org/10.1371/journal. pone. 0177155

22. Dong $\mathrm{Y}, \mathrm{Mo} \mathrm{X}, \mathrm{Hu} \mathrm{Y}$ et al (2020) Epidemiological characteristics of 2143 pediatric patients with 2019 coronavirus disease in China. Pediatrics. https://doi.org/10.1542/peds.2020-0702

23. Kuk JL, Church TS, Blair SN, Ross R (2006) Does measurement site for visceral and abdominal subcutaneous adipose tissue alter associations with the metabolic syndrome? Diabetes Care 29:679-684

Publisher's Note Springer Nature remains neutral with regard to jurisdictional claims in published maps and institutional affiliations. 